Gynäkologe 2010 · 43:203-204

DOI 10.1007/s00129-009-2479-7

Online publiziert: 5. März 2010

(c) Springer-Verlag 2010

\author{
W. Jonat ${ }^{1} \cdot$ R. Kreienberg ${ }^{2} \cdot K$. Diedrich ${ }^{3}$ \\ ${ }^{1}$ Klinik für Gynäkologie und Geburtshilfe, Universität Schleswig-Holstein, Campus Kiel \\ ${ }^{2}$ Universitätsfrauenklinik Ulm \\ ${ }^{3}$ Klinik für Frauenheilkunde und Geburtshilfe, Universitätsklinikum Schleswig-Holstein, \\ Campus Lübeck
}

\title{
Zentrumsbildung in der Gynäkologie und Geburtshilfe
}

Eine der großen Herausforderungen in der Frauenheilkunde und ihren Spezialdisziplinen besteht darin, das Fach bei den bestehenden gesellschaftlichen und gesundheitspolitischen Veränderungen so fortzuentwickeln, dass es für zukünftige Anforderungen gerüstet sein wird. Gynäkologie und Geburtshilfe haben sich zum Begleiter der Gesundheit von Frauen während ihres gesamten Lebens entwickelt. Zunehmend stellt jedoch besonders die älter werdende Gesellschaft für die Frauenheilkunde eine große Herausforderung dar. Unabhängig vom Lebensalter können viele Erkrankungsbilder nur noch in komplexen Behandlungsstrukturen betreut werden, da nur so dem Anspruch auf eine hochwertige Behandlungsqualität Rechnung getragen werden kann.

Die Zielsetzung einer bestmöglichen Qualität hat in vielen Bereichen unseres Fachgebietes zur „Zentrenbildung" geführt. Leider bleibt die politische Unterstützung von Institutionen, die auf exakt definierten Gebieten besondere Qualifikationen und umfassende Erfahrung bieten können, teilweise immer noch hinter den organisatorischen und medizinischen Notwendigkeiten zurück. Beispielhaft sollen in dieser Ausgabe von „Der Gynäkologe" sechs spezialisierte Typen von „Fachzentren“ genannt werden, die bereits heute erheblichen Einfluss auf die Strukturen unseres Fachgebietes haben.

\section{( ) Brustzentren stellen die Vorreiter hoch spezialisierter medizinischer Zentren dar}

In Brustzentren und in gynäkologischen Krebszentren soll die Therapie von Frauen mit Mamma- und Genitalmalignomen leitliniengerecht und qualitätsgesichert erfolgen. Die Etablierung dieser Krebszentren ist Bestandteil des Nationalen Krebsplanes sowie des Strategieplanes der Deutschen Krebsgesellschaft. Die Brustzentren haben sich in Deutschland als erste Organzentren überhaupt strukturiert und stellen damit die Vorreiter hoch spezialisierter medizinischer Zentren dar. Diagnostik, Therapie und Nachsorge binden eine Vielzahl von Fachdisziplinen ein. Krankenhäuser der universitären Maximalversorgung, der Zentralversorgung und der Grund- und Regelversorgung können mit den Niedergelassenen Netzwerke bilden und damit onkologische Qualität auf höchstem Niveau bieten. Neben der intersektoralen Versorgung stellt besonders die interdisziplinäre Versorgung eine Herausforderung dar. Die zertifizierten Brustzentren müssen sich verpflichtend mit der strukturierten Versorgungsoptimierung beschäftigen und für eine transparente Qualitätssicherung sorgen. Die Versorgungskette vom Mammographie-Screening über die interventionelle Diagnostik und die Therapie im Brustzentrum bis hin zur Nachsorge ist zum Teil noch lückenhaft qualitäts- gesichert und sollte weiter optimiert werden. Die Zentralisierung und die externe Zertifizierung stellen sicher, dass Diagnostik wie Therapie des Mammakarzinoms heute mehrheitlich - wie, mit verstärktem Augenmerk, auch die der gynäkologischen Malignome - evidenzbasiert durchgeführt werden. Das Ziel eines deutschlandweiten Netzwerkes von zertifizierten multidisziplinären Brustzentren ist in der Zwischenzeit weitgehend erreicht worden. Dieser Erfolg steht für die gynäkologischen Krebszentren noch aus.

Die eigentlichen Endpunkte in der Krebstherapie, die Langzeitüberlebensrate bzw. die Rezidiv-/Metastasierungsrate, können leider in der Mehrzahl der Zentren bislang nur durch Surrogatparameter ungenau erfasst werden. Dennoch zeigen sich schon jetzt unterschiedliche Trends bei zertifizierten und nicht zertifizierten Brustzentren.

\section{( In zertifizierten \\ Dysplasiezentren sollen \\ Patientinnen nach internationalem Standard behandelt werden}

Wie in anderen Ländern Europas ist auch in Deutschland eine Zertifizierung (durch die Arbeitsgemeinschaft Kolposkopie und Zervixpathologie) als Dysplasiezentrum möglich. Die Zertifizierung setzt eine Mindestzahl an Kolposkopien, die Möglichkeit zur Durchführung emp- 
fohlener Operationstechniken und die Teilnahme an Fortbildungen voraus. Als externe Qualitätskontrolle ist ein Benchmarking verbindlich. Zertifizierte Dysplasiezentren können zu einer Verbesserung der Vorsorge führen und auch sie sollen helfen, mehr Patientinnen nach internationalem Standard zu behandeln.

Auch im Bereich der Geburtshilfe kommt es zu Zentrenbildungen. Die Teams der Perinatalzentren umfassen Perinatalmediziner, Geburtshelfer, Neonatologen sowie Angehörige weiterer, benachbarter Fachgebiete. Bisher wurden die Perinatalzentren in Deutschland nach Strukturvorgaben definiert. Ab 2010 kommen relativ niedrige Behandlungsmindestmengen für kleine Frühgeborene und kranke Neugeborene hinzu. Eine Zertifizierung nach Art der onkologischen Zentren ist kurzfristig nicht vorgesehen.

Auch die Beckenbodenzentren (Kontinenzzentren durch DKS, Deutsche Kontinenzgesellschaft)wollen durch ihre Etablierung und Zertifizierung der optimierten interdisziplinären Zusammenarbeit der beteiligten ärztlichen und nichtärztlichen Fachbereiche dem Wohl der Patientinnen dienen. Die Zentren beschäftigen sich schwerpunktmäßig mit funktionellen Störungen und Erkrankungen des Beckenbodens. Voraussetzung für die interdisziplinäre Zertifizierung sind strukturelle und medizinisch-inhaltliche Kriterien.

Ein ähnliches Ansinnen haben Endometriosezentren, die eine strukturelle Voraussetzung für eine verbesserte Diagnostik und Therapie der Endometriose schaffen sollen. Die Zertifizierung erfolgt durch externes Audit nach definierten Kriterien durch die Stiftung EndometrioseForschung zusammen mit der Endometriose-Vereinigung e.V. und der Europäischen Endometriose-Liga. Neben der multimodalen Behandlung müssen die Zentren eine suffiziente Ausbildung des medizinischen Personals leisten und durch Information der Bevölkerung und Unterstützung der Selbsthilfegruppen das Verständnis für die Erkrankung verbessern.

Die Implementierung der „Zentren“ in den unterschiedlichen Subspezialitäten hat positive und negative Aspekte. Selbsthilfegruppen und politische Gremien be- grüßen sie als den richtigen Weg zur Verbesserung der Versorgungsstrukturen. Einige Kollegen sind jedoch in Sorge, dass sie einen Imageverlust bei fehlender Zertifizierung erleiden und von den weiteren Entwicklungen ausgeschlossen werden könnten. Während Struktur- und Prozessqualität, besonders der onkologischen Zentren, gut überprüfbar sind, fehlt bisher in Deutschland die Möglichkeit, die Ergebnisqualität der genannten Zentren direkt zu messen und zu vergleichen. Dieses Manko muss kurzfristig behoben werden, da als Nachweis für eine optimierte Versorgung in der Medizin eine nachweisbar gute Ergebnisqualität unverzichtbar ist.

Nicht nur vor dem Hintergrund knapper finanzieller Ressourcen ist eine Fokussierung auch im Bereich der Gynäkologie und Geburtshilfe aus verschiedenen Gründen absehbar und notwendig. So wird ein wesentliches Problem der Zukunft die qualifizierte Weiterbildung in den Schwerpunkten und weiteren Subspezialisierungen sein. Zukünftig wird sich Nachwuchs dort bewerben, wo eine zeitnahe und kompetente Ausbildung möglich ist. Nur eine ausreichende Anzahl qualifizierter Zentren mit hohen $\mathrm{Pa}$ tientinnenzahlen kann zukünftig die notwendigen Nachwuchskolleginnen und -kollegen ausbilden. Auch die Deutsche Gesellschaft für Gynäkologie und Geburtshilfe hat die qualifizierte Aus-, Fortund Weiterbildung junger Ärztinnen und Ärzte als eins ihrer wichtigsten Anliegen benannt. Dieses wurde insbesondere mit der Gründung der Deutschen Akademie für Gynäkologie und Geburtshilfe unterstrichen.

Die Bildung von spezialisierten interdisziplinären Zentren in unterschiedlichen Bereichen der Frauenheilkunde und Geburtshilfe führt zu großen Umwälzungen in unserem Fach. Neben der qualifizierten Versorgung selektierter Patientengruppen sind die Zentren für die Vermittlung spezifischer Fortbildungsinhalte sicherlich vorteilhaft. Auch aus volkswirtschaftlicher Sicht könnten sich Vorteile und erhebliche Einsparmöglichkeiten dadurch ergeben, wenn nicht mehr alle medizinischen Optionen überall angeboten würden. Allerdings erscheint es dringend notwendig, darauf zu achten, die Einheit des Faches der Gynäkologie und Geburts- hilfe zu erhalten und seine Konturen weiter zu schärfen. Nur ein starkes, vereintes Fach wird weiterhin in der Lage sein, tatsächlich der maßgebliche gesundheitliche Begleiter der Frauen während des gesamten Lebens zu sein.

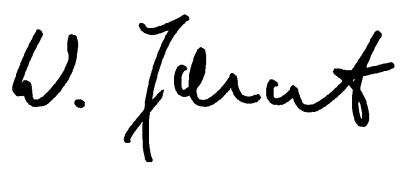

Prof. Dr. W. Jonat

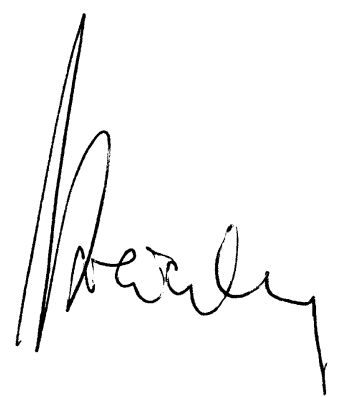

Prof. Dr. R. Kreienberg

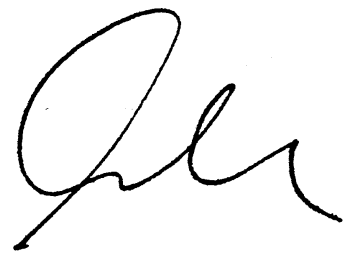

Prof. Dr. K. Diedrich

\section{Korrespondenzadresse \\ Prof. Dr. Dr. h.c. W. Jonat}

Klinik für Gynäkologie und Geburtshilfe, Universität Schleswig-Holstein, Campus Kiel Arnold-Heller-Str. 3, 24105 Kiel

jonat@email.uni-kiel.de 